Supporting Information

\title{
Co-delivery of Chemotherapeutic Drugs and Immune Adjuvants by Nanoscale DNA Tetrahedrons for Synergistic Cancer Therapy
}

Qian Wang ${ }^{1,2, \ddagger}$, Yuxuan Ma ${ }^{1,2, \ddagger}$, Zhangwei $L u^{1,2}$, Hanyang $Y^{1,3,4}$, and Zhe $L i^{1,2, *}$

${ }^{1}$ Department of Biomedical Engineering, College of Engineering and Applied Sciences, Jiangsu

Key Laboratory of Artificial Functional Materials, Nanjing University, Nanjing, Jiangsu 210023, China

${ }^{2}$ State Key Laboratory of Analytical Chemistry for Life Science, Nanjing University, Nanjing, Jiangsu 210023, China

${ }^{3}$ State Key Laboratory of Coordination Chemistry, Nanjing University, Nanjing, Jiangsu 210023, China

${ }^{4}$ Chemistry and Biomedicine Innovation Center (ChemBIC), Nanjing University, Nanjing, Jiangsu 210023, China

${ }^{5}$ School of Chemistry and Chemical Engineering, Nanjing University, Nanjing, Jiangsu 210023, China

*Corresponding Author

zheli@nju.edu.cn 


\section{Materials and methods}

\section{Materials.}

DNA oligonucleotides were purchased from Genscript (Nanjing, China) and their sequences were listed in Table S1. Red blood cell lysis buffer and DOX were purchased from Sangon

Biotechnology (Shanghai, China). Elisa kits were purchased from FcmacsBiotechCo., Ltd (Nanjing, China). Antibodies were purchased from Multiscience Biotech, Co., Ltd (Hangzhou, China). Human L02 cells, mouse J744A.1 cells and 4T1 cells were purchased from Mingjin Biology (Shanghai, China).

\section{Preparation and characterization of DNA nanocarriers.}

DNA tetrahedrons without/with CpG (DTN/ DTN-CpG) were prepared as described before.

Briefly, strands were mixed at the designed ratios (T-A, T-B, T-C, T-D, AS1411-A, random-B, random-C random-D for DTN; T-A, T-B, T-C, T-D, AS1411-A, CpG-B, CpG-C, CpG-D for DNT-CpG; $2 \mu \mathrm{M}$ each strand) in $1 \mathrm{x} \mathrm{TE} \cdot \mathrm{Mg}^{2+}$ buffer and annealed using a PCR machine (Eppendorf Mastercycler nexus, Germany). The construction of the structures was analyzed by Native PAGE.

\section{DOX loading.}


DOX $(1 \mathrm{mM})$ was incubated with DNA tetrahedron $(1 \mu \mathrm{M})$ for $2 \mathrm{~h}$ at room temperature and centrifuged at 2,000g for 30 seconds. After centrifuging, the free DOX in the supernatant and DTN/DOX or DTN-CpG/DOX complex in the red precipitate were isolated. The absorption of the supernatant at $480 \mathrm{~nm}$ (named $\mathrm{A}_{\text {sup }}$ ) was measured to quantify the unloaded DOX. The absorption

of $1 \mathrm{mM}$ DOX incubated with $1 \mathrm{x} \mathrm{TE} \cdot \mathrm{Mg}^{2+}$ buffer as control was named $\mathrm{A}_{\mathrm{ctrl}}$. And the loading efficiency was calculated as below.

$$
\text { The loading efficiency of } \frac{D T N}{D O X}=\left(1-\frac{A_{\text {sup }}}{A_{c t r l}}\right) \times 100 \% \#(1)
$$

The DTN/DOX or DTN-CpG/DOX complex in the red precipitate was then redissolved in PBS for further experiment.

\section{Characterization of DTN-CpG/DOX.}

AFM imaging was performed on a Bruker Multimode 8 with SNL-10 tips in ScanAsyst mode.

The diameter and zeta potential were determined by DLS (Malvern Zetasizer Nano ZS90, UK).

\section{Fluorescence microscopy.}

4T1 cells were seeded on confocal dishes and cultured overnight. Cy5-labeled DTN was incubated with DOX and purified through centrifuge and then added into cell culture for $3 \mathrm{~h}$. The 
nuclei were stained using DAPI or Hoechst 33258. Imaging was performed on a laser confocal

fluorescent microscope (Olympus FV3000, Janpan)

\section{Cell viability assay.}

Annexin V-FITC/PI apoptosis detection kit (Beyotime Biotechnology) and cell-counting kit-8

(cck-8, Dojindo) were used to demonstrate the cytotoxicity of DTN/DOX.

\section{Cytokine assays.}

$1 \times 10^{6}$ J744A. 1 cells were seeded and incubated overnight. Then $100 \mathrm{nM} \mathrm{CpG} \mathrm{ODNs} \mathrm{or} 100$ nM DTN with various numbers of $\mathrm{CpG}$ from 0 to 3 were added in the medium. After the incubation procedure of 24 hours (IL-6) or 12 (TNF- $\alpha$ ) hours, the concentrations of cytokine in the medium were determined by ELISA.

\section{T1 tumor-bearing mice model.}

Female BALB/c mice (6-7 weeks old, 17-19 g) were purchased from Cavens Laboratory Animal Ltd. (Changzhou, China). The 4T1 tumor-bearing mice model was established by injecting $1 \times 10^{6}$ 4T1 cells dispersed in PBS into left upper flanks of BALB/c mice. All animal procedures were in accordance with the guidelines of the Institutional Animal Care and Use Committee.

\section{Tumor growth inhibition.}


Tumor-bearing mice were divided into 4 groups ( $\mathrm{n}=5$ per group) including PBS, DOX, DTNCpG/DOX. The mice were administered with equivalent doses (DTN-CpG: $0.75 \mathrm{mg} / \mathrm{kg}$, DOX: 2 $\mathrm{mg} / \mathrm{kg}$ ) via tail vein injection in $100 \mathrm{~mL}$ volume every three days for 5 treatments. After 15 days of treatments, the mice were sacrificed. Major organs and the tumor tissue were harvested for further experiments including HE stain, immunofluorescence, and immunohistochemistry. The weight of the mice was measured before each injection.

\section{Levels of TNF- $\alpha$ in the serum.}

After one day of treatment, the serum samples of mice in all the groups were collected. Then the levels of TNF- $\alpha$ in the serum were determined by ELISA.

\section{Flow cytometry assay.}

Single cells were isolated from spleen and then incubated with anti-CD4-FITC, anti-CD8a-PE, and anti-CD3e-APC. FACS analysis was performed on a FACSVerse (BD Verse, USA). 
Table S1. Sequences of DNA oligonucleotides.

\begin{tabular}{|c|c|}
\hline Name & Sequence \\
\hline $\mathrm{T}-\mathrm{A}$ & $\begin{array}{l}\text { ACATTCCTAAGTCTGAAACATTACAGCTTGCTACACGAGAAGAGCCGCCATA } \\
\text { GTAACATCCCGCTTGAAGTGC }\end{array}$ \\
\hline T-B & $\begin{array}{l}\text { TATCACCAGGCAGTTGACAGTGTAGCAAGCTGTAATAGATGCGAGGGTCCA } \\
\text { ATACTCTCTTCACCGTAATCTT }\end{array}$ \\
\hline $\mathrm{T}-\mathrm{C}$ & $\begin{array}{l}\text { TCAACTGCCTGGTGATAAAACGACACTACGTGGGAATCTACTATGGCGGCTC } \\
\text { TTCAGCAACCTGCCTGTTAGC }\end{array}$ \\
\hline T-D & $\begin{array}{c}\text { TTCAGACTTAGGAATGTGCTTCCCACGTAGTGTCGTTTGTATTGGACCCTCGC } \\
\text { ATTACCTGATCGTCTGGTCA }\end{array}$ \\
\hline AS1411 & GGTGGTGGTGGTTGTGGTGGTGGTGGTTTTTTGCACTTCAAGCGGGATGT \\
\hline CpG-B & TCCATGACGTTCCTGACGTTTTTTAAGATTACGGTGAAGAGA \\
\hline CpG-C & TCCATGACGTTCCTGACGTTTTTTGCTAACAGGCAGGTTGCT \\
\hline CpG-D & TCCATGACGTTCCTGACGTTTTTTTGACCAGACGATCAGGTA \\
\hline Random-B & ATAATAATAATAATAATATTTTTTAAGATTACGGTGAAGAGA \\
\hline Random-C & ATAATAATAATAATAATATTTTTTGCTAACAGGCAGGTTGCT \\
\hline Random-D & ATAATAATAATAATAATATTTTTTTGACCAGACGATCAGGTA \\
\hline
\end{tabular}



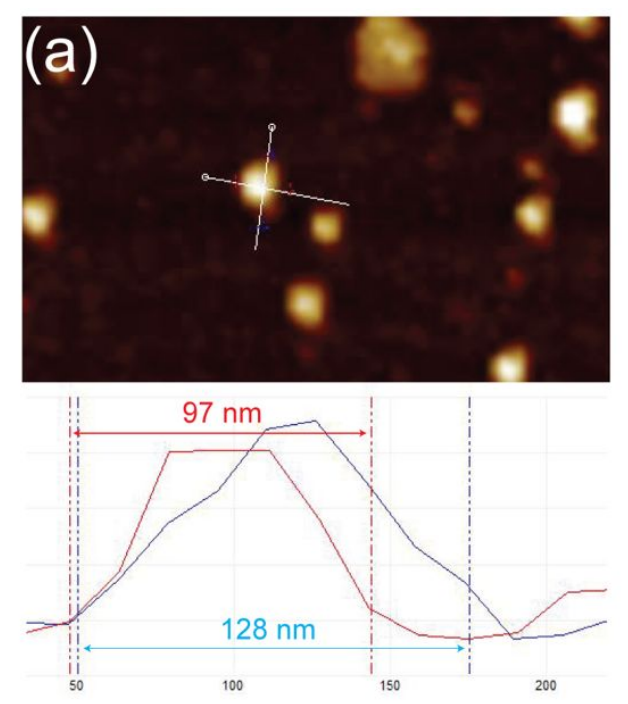

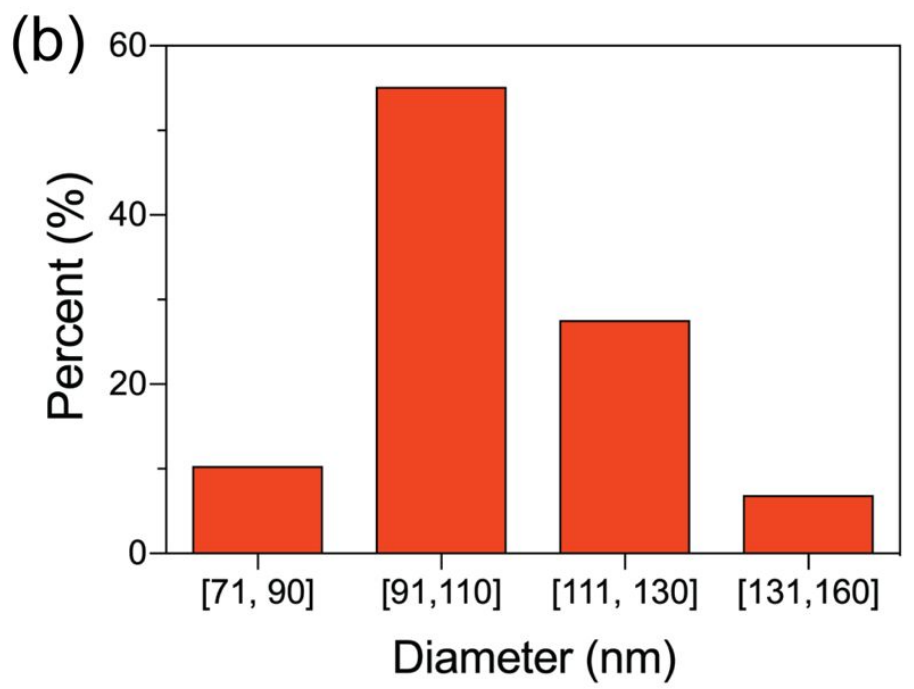

Figure S1. The particle dimensions measured by AFM (a) and corresponding size distribution (b). The average diameter was about $106 \mathrm{~nm}$.
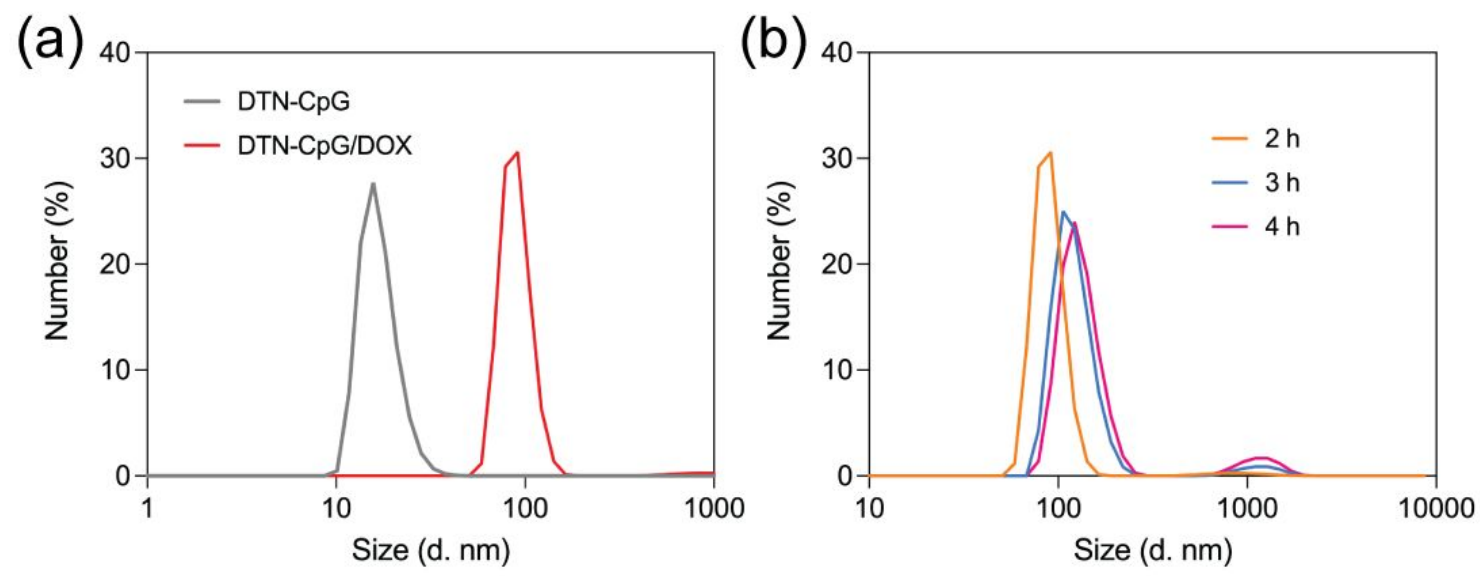

Figure S2. Hydrodynamic diameter by DLS analysis of (a) DTN-CpG and DTN-CpG/DOX, (b) DNA tetrahedron and DOX complex incubated for 2, 3 and 4 hours. 
Zeta Potential Distribution

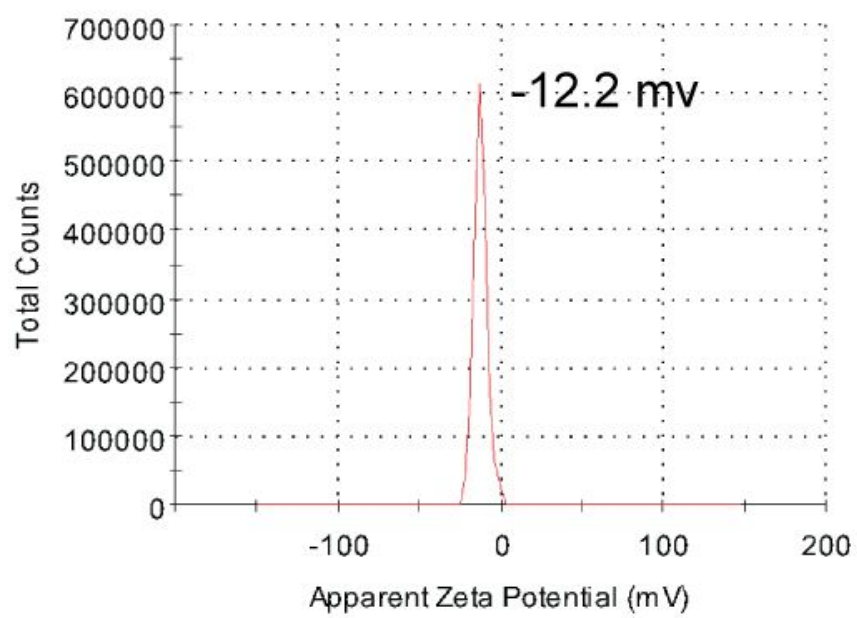

Figure S3. Zeta potential of DTN-CpG/DOX.

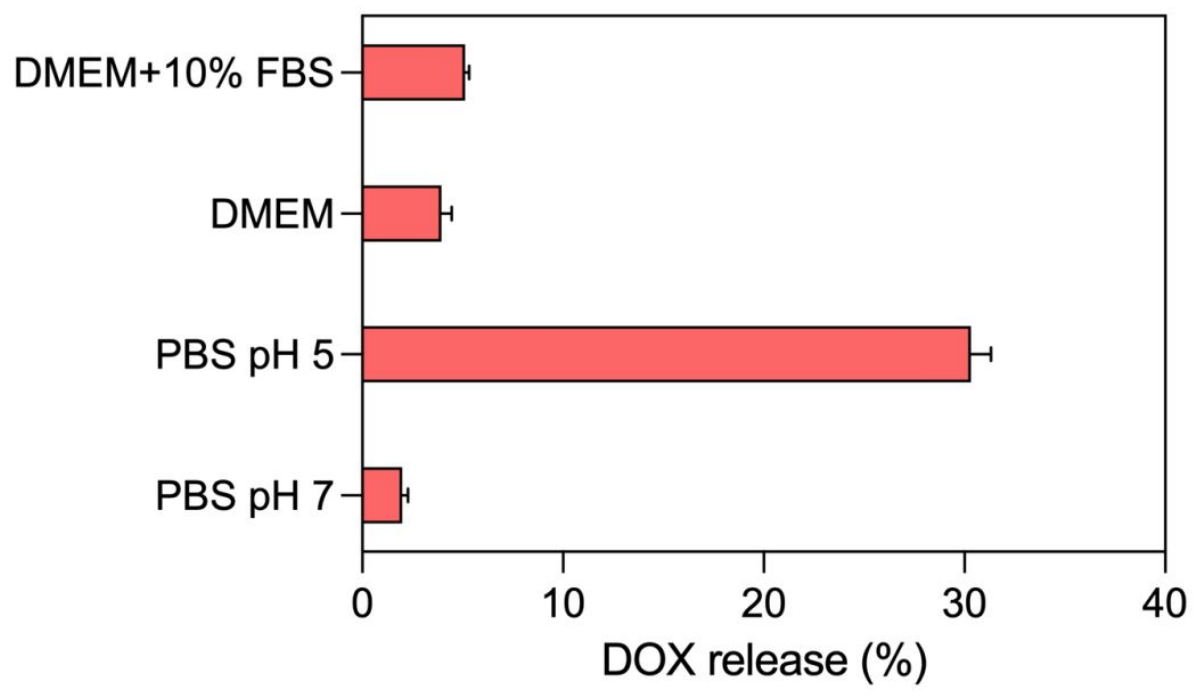

Figure S4. The study of DOX release on DTN-CPG/DOX under different conditions. DOX could be released more easily under acidic conditions. 
(a)

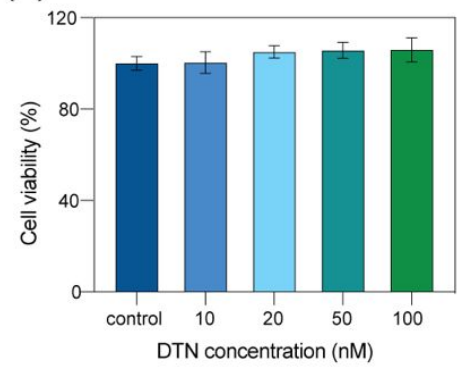

(b)

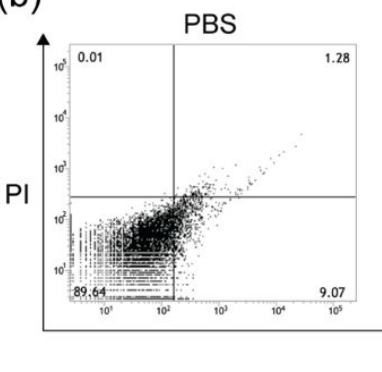

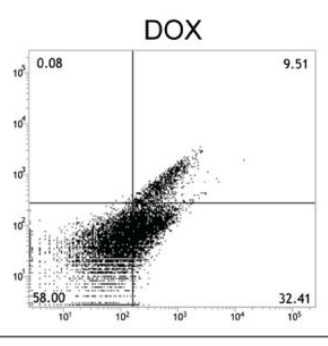

Annexin V

Figure S5. Cytotoxicity of DTN on 4T1 cell. (a) MTT assay of cell viability showed that DTN had no cytotoxicity on 4T1 cells at the DTN concentrations up to $100 \mathrm{nM}$. (b) Cell apoptosis of 4T1 cells incubated with different conditions determined by flow cytometry analysis. The DNA tetrahedron showed no notable influence on the efficacy of DOX to 4T1 cancer cells.

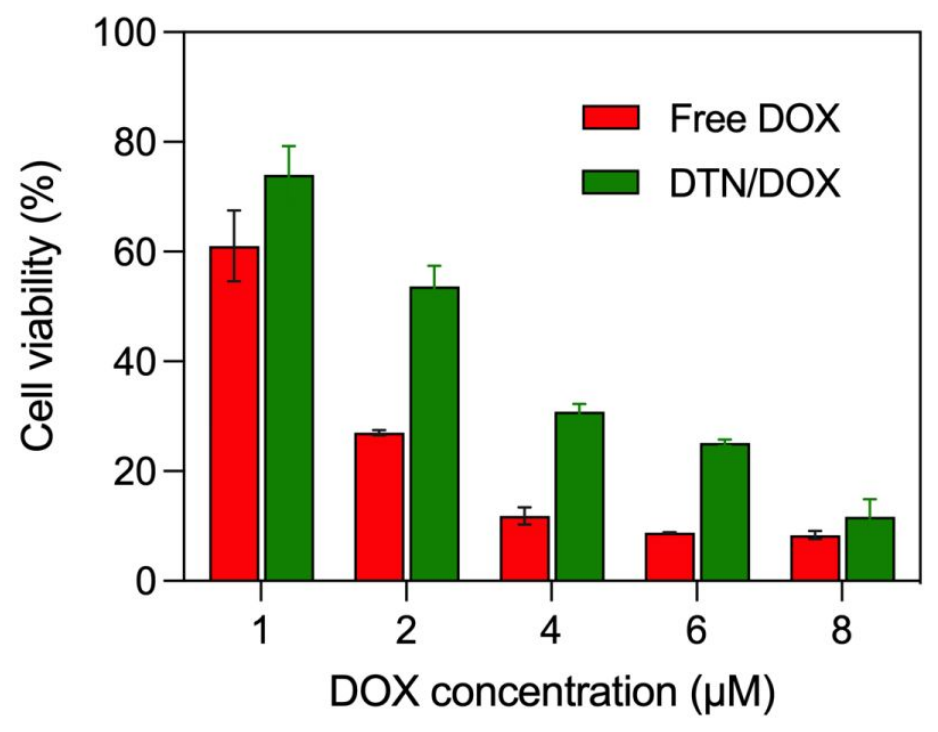

Figure S6. MTT assay of normal liver L02 cell line treated with free DOX and DTN/DOX at the DOX concentrations up to $8 \mu \mathrm{M}$. DTN reduced the toxicity of DOX to normal cells. 

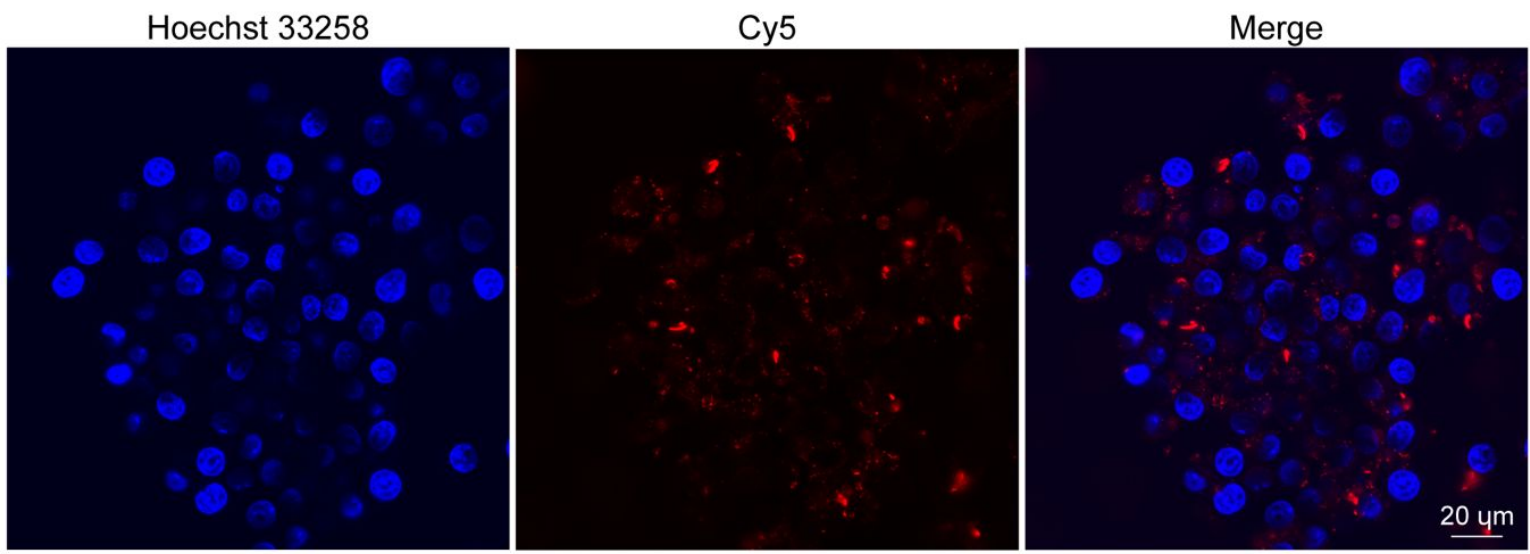

Figure S7. Confocal images of J744A.1 cells after incubation with Cy5-DTN-CpG for $3 \mathrm{~h}$, demonstrating that DTN can deliver $\mathrm{CpG}$ into macrophages.

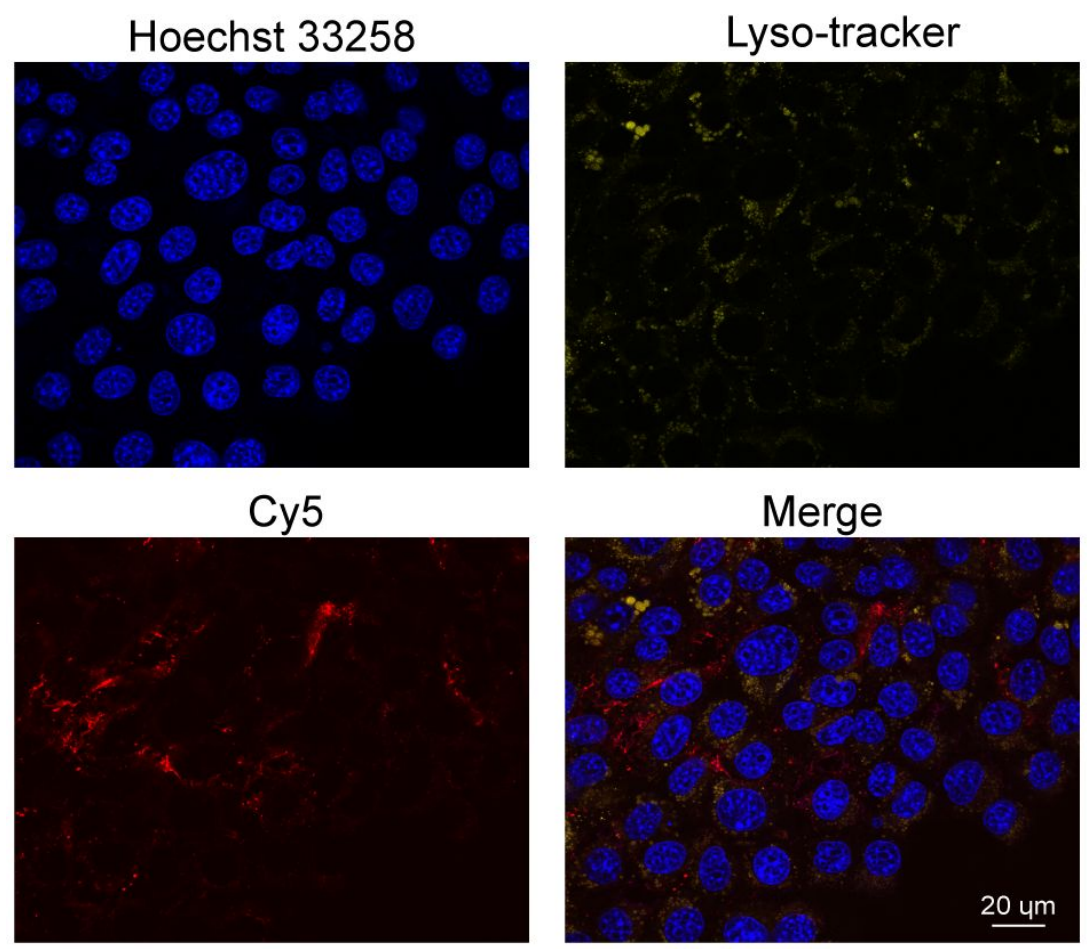

Figure S8. Confocal images of 4T1 cells after incubation with Cy5-DTN-CpG/DOX for 4 h. No overlapping fluorescence between lysosome and DTN-CpG was observed, indicating that DTN$\mathrm{CpG}$ could escape from the lysosome. 

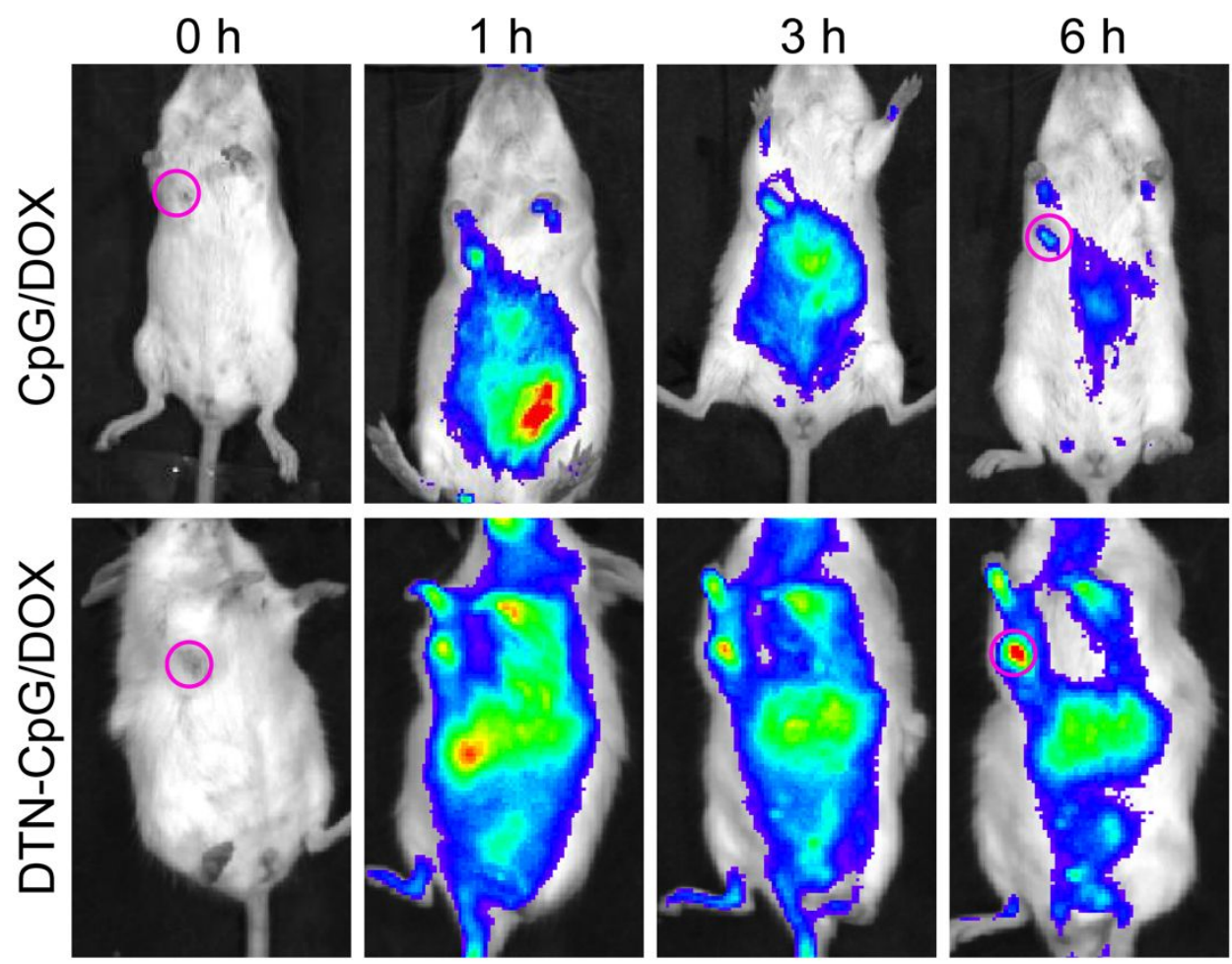

Figure S9. Dynamic biodistribution of Cy5.5-CpG/DOX, Cy5.5-DTN-CpG/DOX in the tumorbearing mice through fluorescence imaging. DTN-CpG/DOX demonstrated a much stronger signal in the tumor region than $\mathrm{CpG} / \mathrm{DOX}$. 

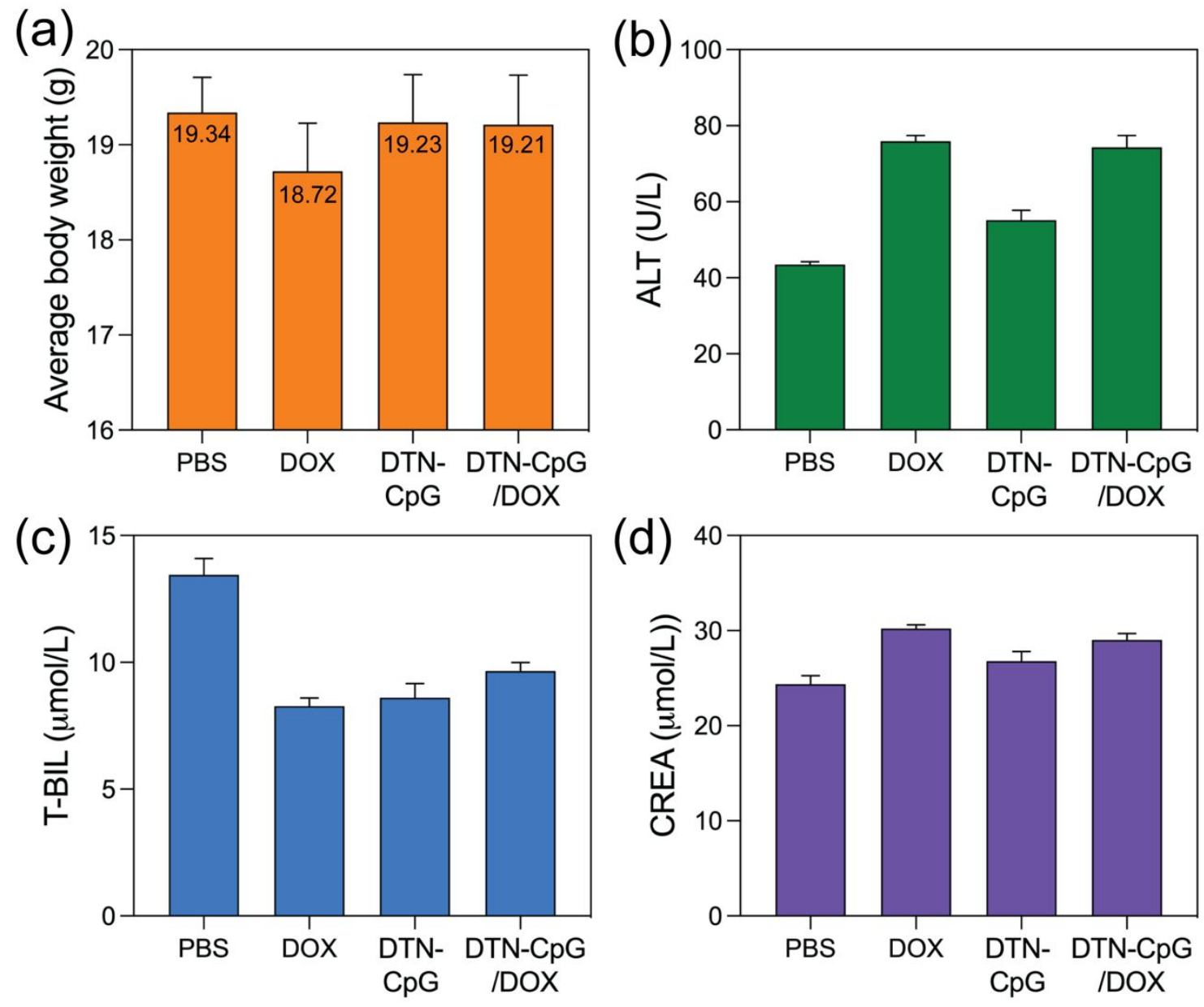

Figure S10. Average body weight, serum biochemical and hematological parameters of mice after treatments. (a) After the 15-day treatment, the body weight of the mice in the DOX group was slightly lower than that of the other groups. The levels of (b) alanine aminotransferase (ALT), (c) total bilirubin (T-Bil) and (d) creatinine (CREA) of treatment groups were slightly different from those of the control group, but within the normal reference range. 

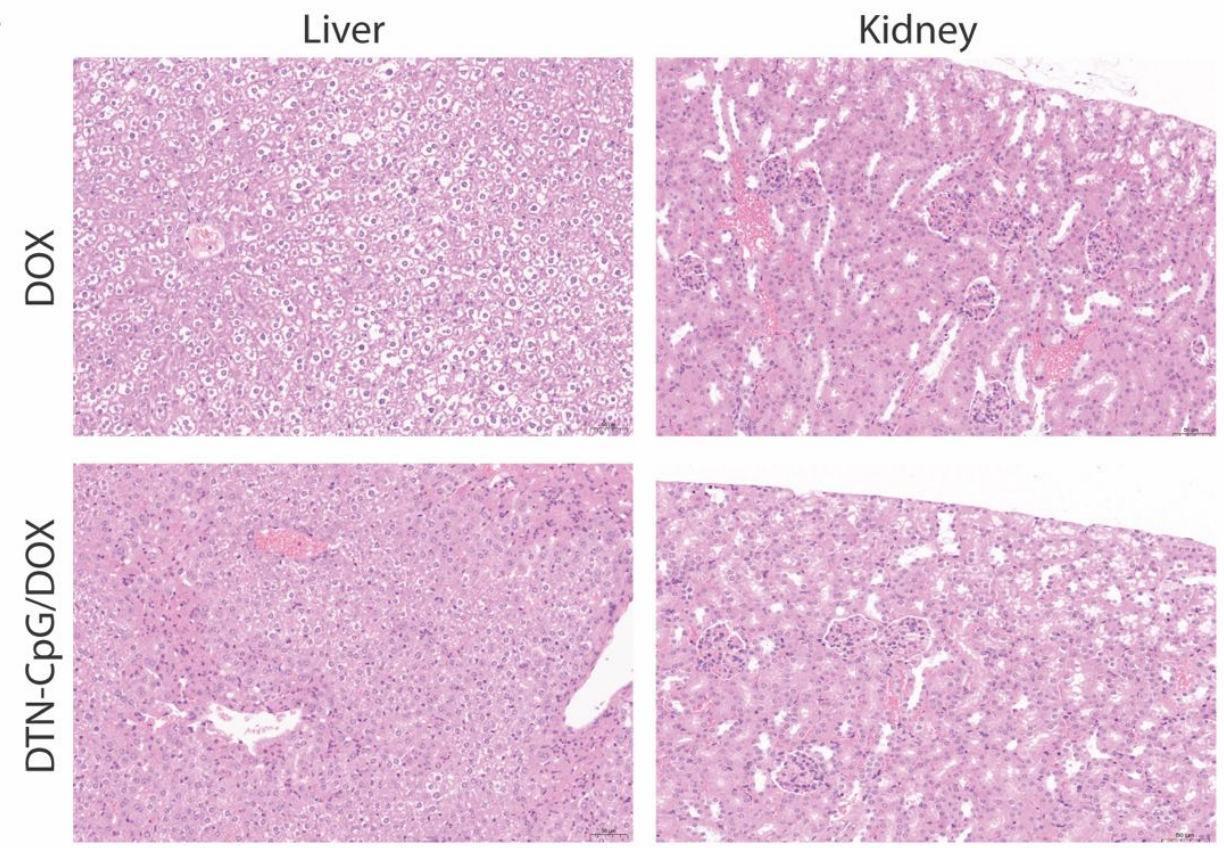

Figure S11. H\&E staining results of liver and kidney of mice with a double-dose injection. The liver cells in the DOX group exhibited more severe vacuolar degeneration than those in DTN$\mathrm{CpG} / \mathrm{DOX}$ group, suggesting that DTN-CpG/DOX could reduce the systemic toxicity of DOX. 


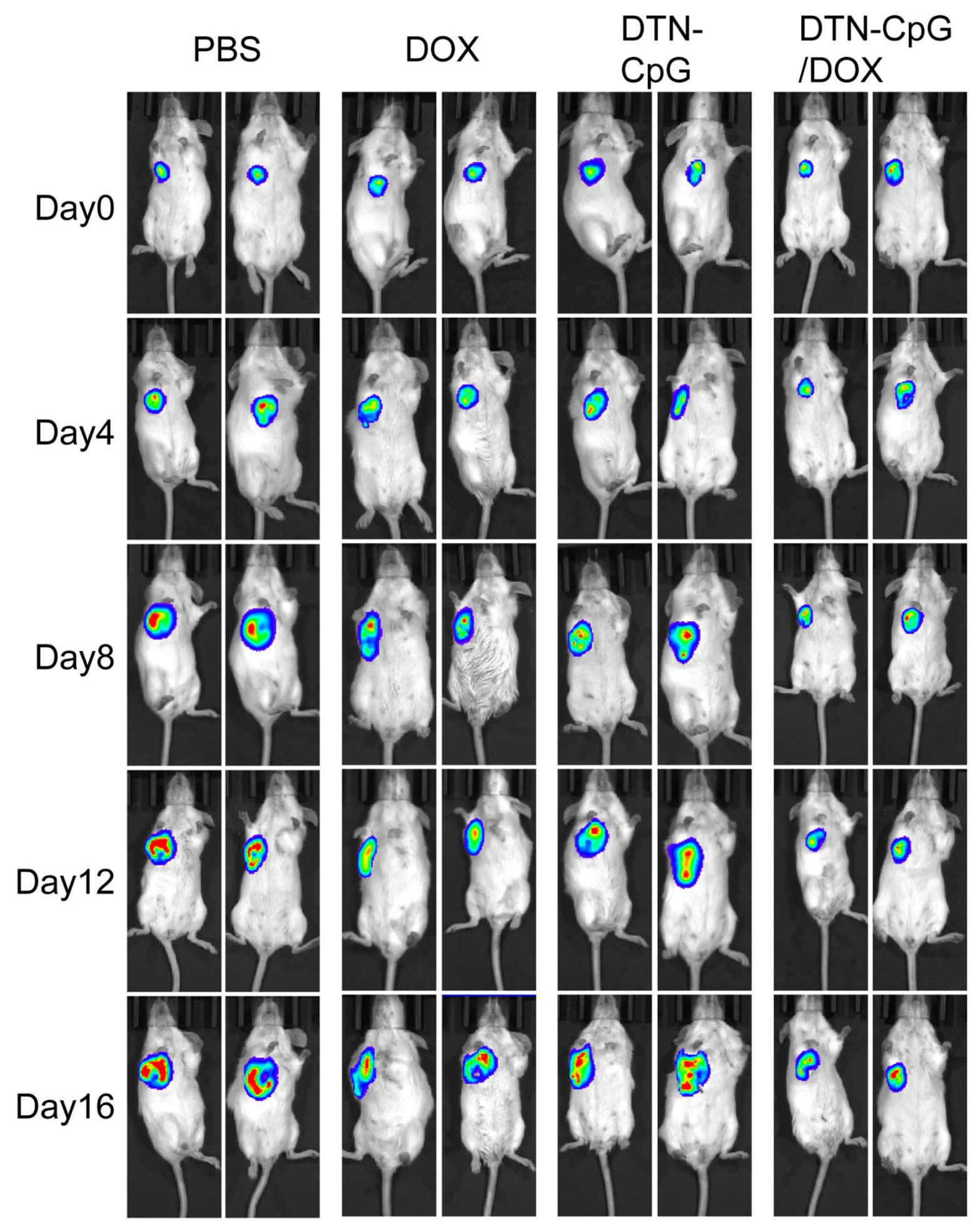

Figure S12. The tumor size change during the treatments. The DTN-CpG/DOX group showed the best anti-tumor effect than single chemotherapy or immunotherapy. 


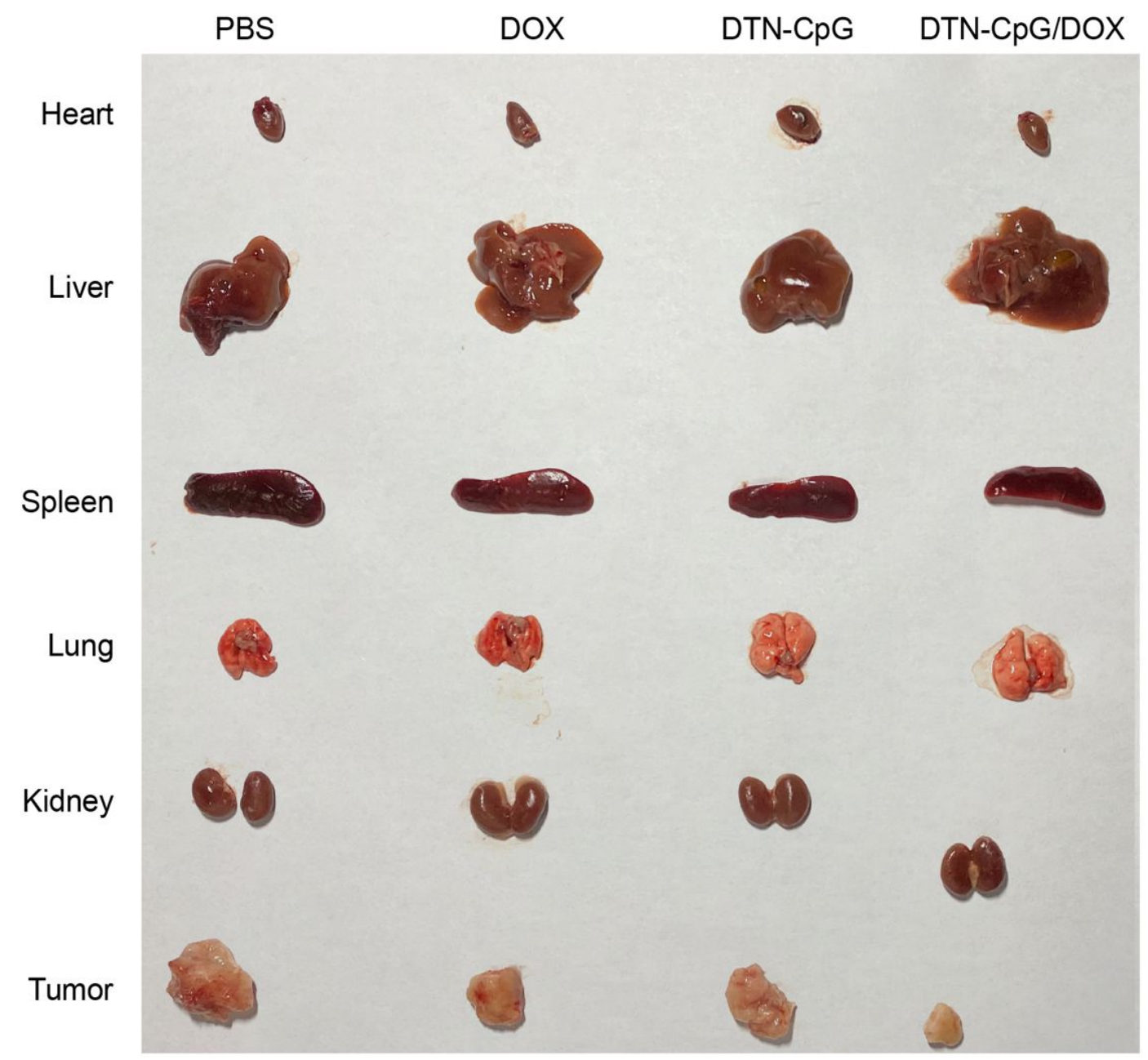

Figure S13. Photographs of major organs and tumor tissues. The DTN-CpG/DOX group showed the best anti-tumor efficiency. 


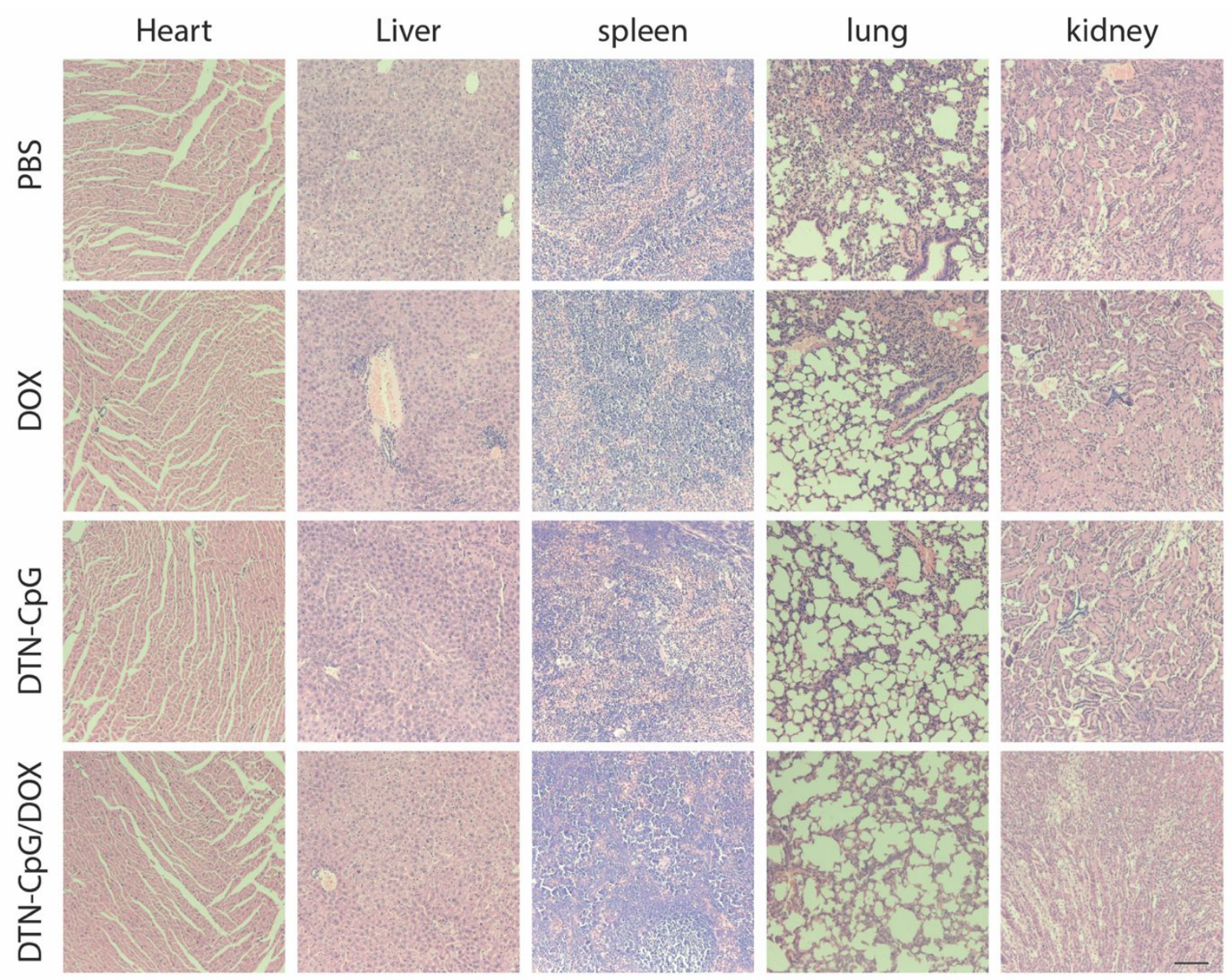

Figure S14. H\&E staining results of major organs. Scale bar, $100 \mu \mathrm{m}$. No adverse effects on major organs were observed, indicating the excellent biocompatibility of the delivery system for in vivo applications. 